\title{
Bone Seeking Radiopharmaceuticals for Metastatic Bone Pain
}

\author{
Marnix G.E.H. Lam ${ }^{1}$ and Bernard A. Zonnenberg ${ }^{1,2}$ \\ ${ }^{1}$ Department of Radiology and Nuclear Medicine \\ ${ }^{2}$ Department of Internal Medicine \\ University Medical Center Utrecht \\ The Netherlands
}

\section{Introduction}

Prostate cancer is the most common malignancy in men in the Netherlands. The majority of patients with advanced prostate cancer develop skeletal metastases, with bone pain as a frequent symptom. This poses a significant medical problem. Amongst other treatments, bone seeking radiopharmaceuticals are used to palliate painful metastatic bone disease. Efficacy has been sufficiently proven, as well as safety and feasibility. Practical issues as well as new developments and future prospects will be discussed.

This chapter will discuss the treatment of metastatic bone pain in hormone-refractory prostate cancer patients with bone seeking radiopharmaceuticals. Two main issues will be discussed:

1. Bone seeking radiopharmaceuticals in clinical practice. Issues regarding radiation safety and a protocol for routine use of bone seeking radiopharmaceuticals will be discussed in order to improve routine clinical care.

2. Enhancement of efficacy of bone seeking radiopharmaceuticals.

But first a short introduction will be given on clinical relevance and bone seeking radiopharmaceuticals in general.

\section{Prostate cancer and skeletal metastases}

The incidence of malignancy in the Netherlands was 74.500 patients in 2005. This will increase to approximately 95.000 new cases in 2015. Because malignancy related death is decreasing and it is likely to decrease further the prevalence of cancer patients will increase to an estimated 692.000 patients in 2015, compared to 366.000 in 2000 (an estimated doubling time of 15 years) (Coebergh, van de Poll-Franse, and Alers 2004; Visser and van Noord 2005). One of the major causes of cancer related death in men is prostate cancer. These patients will be the focus of this chapter.

The incidence of prostate cancer is high worldwide. It is the most common malignancy in men in the Netherlands. Approximately 9000 men are being diagnosed with prostate cancer each year (Figure 1). The rising incidence may be attributed to the incremental use of screening methods using prostate specific antigen (PSA) to detect prostate cancer. This hypothesis is supported by the growing number of patients being diagnosed with early 
stages of prostate cancer. The incidence also increases with age. Consequently, with a growing number of old men in our society the incidence of prostate cancer will further increase. Fortunately mortality from prostate cancer is decreasing due to better diagnostic methods and treatments (Dijkman and Debruyne 1996).

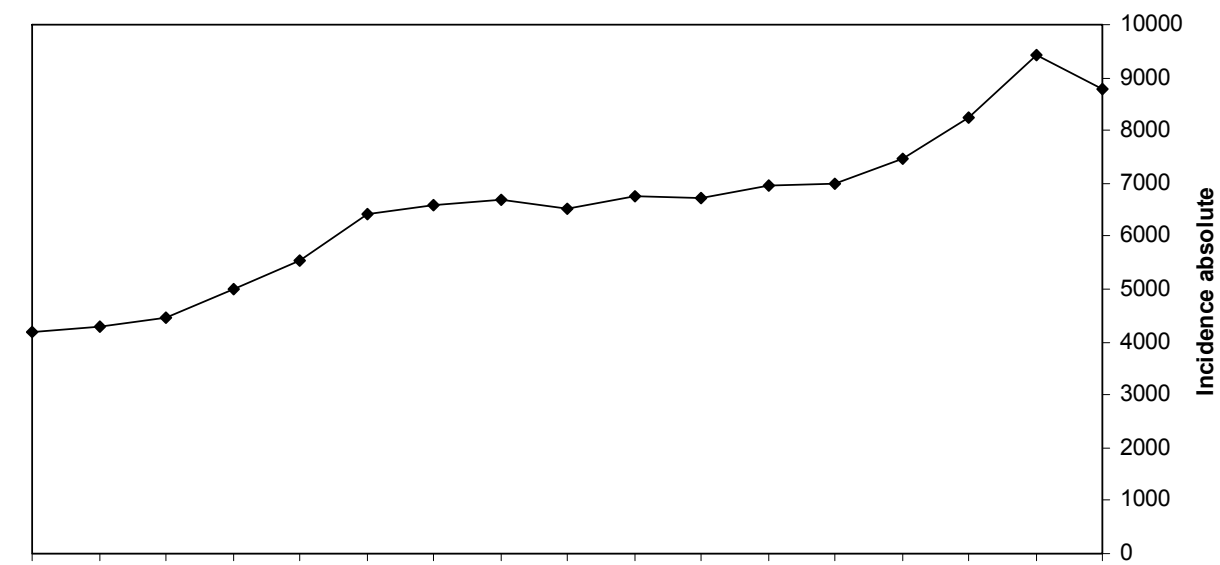

$19891990199119921993199419951996 \quad 1997 \quad 1998 \quad 1999200020012002200320042005$

Years

Fig. 1. Prostate cancer incidence between 1989 - 2005 derived from data on www.ikcnet.nl

A hallmark of metastatic prostate cancer is the development of osteoblastic bone metastases. Almost all patients with advanced prostate cancer eventually develop osseous metastases. In a majority of patients with prostate cancer, bone is the only site of clinical metastases. Many of the established prognostic factors for advanced prostate cancer (eg, performance status, alkaline phosphatase level, and haemoglobin level) reflect the clinical consequences of bone metastases. Hence, patients who develop widespread, progressive, or early bone metastases tend to suffer more from their symptoms and fare worse. Conversely, patients who develop limited, stable, or delayed bone metastases tend to experience less morbidity and have a less dismal clinical outcome. Conceivably, targeting the relevant bone metastases-associated factors may improve therapeutic results (Tu and Lin 2008). These factors consist of the main cells involved in bone metastasis (cancer cells, osteoblasts, osteoclasts, endothelial cells and stromal cells) and the numerous communicating substances (eg, interleukins, VEGF, RANKL, TNF-alfa, endothelins). They form a complex interaction and a microenvironment in which cancer cells may flourish (Tu and Lin 2008).

Frequently these skeletal metastases cause pain. Less common complications include myelum compression and pathological fractures (Dijkman and Debruyne 1996). Besides hormonal treatment most other treatment modalities in advanced prostate cancer patients are intended to palliate bone pain. Metastatic bone pain is a nociceptive somatic pain, initiated and maintained through local tissue injury. It is well recognized that chronic pain (including cancer pain) is a multidimensional phenomenon consisting of five 
dimensions (pathophysiological, sensory, emotional, cognitive, and behavioural). These five dimensions together form a complex pattern of relations (Quirijnen et al. 1996). Stress is a well known stimulus for nociceptive pain. The impression that pain may be uncontrollable produces stress for patients, which may lead to increased suffering and despair, and decreases the patient's performance (Chapman and Gavrin 1999). An effective pain management strategy requires breaking off this cycle using all available means. An effective pain control strategy, however, requires patients to take large quantities of opioids, often as much as $60-200 \mathrm{mg} /$ day. This large dose may cause considerable side effects, including nausea, vomiting, constipation, and central sedation, all of which combine to a decrease in quality of life. Patients will have to take large doses of anti-emetics and laxatives to counteract nausea and constipation, respectively. Central sedation increases drowsiness, resulting in frequent falls, bone fractures, and driving accidents (Etches 1999). Supplemental therapy with local radiation, wide-field radiation, bisphosphonates, or bone seeking radiopharmaceuticals can significantly reduce the dose of opioids for most patients or may even completely eliminate the need for the medications in a few patients (Krishnamurthy and Krishnamurthy 2000).

Metastatic disease in prostate cancer may be treated first with hormonal therapy such as bilateral orchidectomy or medical first line hormone treatment (luteinizing hormone-releasing hormone agonist therapy). This androgen-deprivation therapy may be extended to maximal androgen blockade by adding anti-androgens (bicalutamide, flutamide, nilutamide). High risk prostate cancer patients may benefit from such a regimen even in the early stages of the disease (Klotz 2008). It is also recognized that discontinuation of an anti-androgen once hormone-refractory biochemical progression occurs is associated with a biochemical response in many patients. Anti-androgens may become agonistic due to a combination of androgen receptor over-expression and mutation (Kelly and Scher 1993; Small and Srinivas 1995). After a median of two years the prostate cancer cells generally become insensitive for hormonal treatment. In the case of hormone-refractory disease the patient may be treated by chemotherapy, local radiotherapy, systemic radiopharmaceuticals, bisphosphonates and analgesics, depending on the clinical status (Auclerc et al. 2000).

The clinical benefit of chemotherapy in hormone-refractory prostate cancer patients is limited. Some drugs showed potential as first-line treatment in hormone-refractory prostate carcinoma but were not sufficiently tested in clinical trials (Berthold, Sternberg, and Tannock 2005). Patients however may benefit from docetaxel chemotherapy in combination with prednisone. In a landmark study treatment with $75 \mathrm{mg} / \mathrm{m}^{2}$ docetaxel i.v. every three weeks with $5 \mathrm{mg}$ prednisone twice daily p.o. was compared with mitoxantrone $12 \mathrm{mg} / \mathrm{m}^{2}$ every three weeks (Tannock et al. 2004). The median survival increased from 16.3 months in the mitoxantrone-group to 19.2 months in the docetaxel-group (Berthold et al. 2008). The group receiving docetaxel three weekly had a hazard ratio for death of 0.79 (95 percent confidence interval, 0.67 to $0.93 ; p=0.004$ ) compared to the mitoxantrone-group (Berthold et al. 2008). Pain and quality of life improved significantly better in the docetaxel group and more patients $(45 \%$ versus $32 \%$; $<<0.001)$ showed a $50 \%$ reduction of serum PSA levels (Tannock et al. 2004). However, docetaxel nor any other treatment will be curative in an advanced stage of prostate cancer.

Patients with hormone-refractory prostate cancer who have progressive disease after firstline chemotherapy may still benefit from several treatment options. At this stage of disease, patients can expect only a short duration of survival, and most patients become 
symptomatic. Most patients will cease docetaxel treatment because of progressive disease or unacceptable adverse events. To control symptoms after the cessation of chemotherapy should rely on optimizing medical therapy for palliation. This may be combined with radiotherapy applied to dominant painful bone lesions. External beam radiotherapy for painful skeletal metastases leads to a decrease of pain in $60-65 \%$ of the patients. In $33 \%$ of the patients a total remission of pain symptoms was observed (McQuay et al. 2008). Patients may be treated in one fraction (8 Gray). No difference has been found between such a singledose regimen and multiple fractions (Hartsell et al. 2005; Kaasa et al. 2006; Roos et al. 2005). Furthermore, when needed, patients may be treated a second time with a reported response rate between 66\% and 84\% (Mithal, Needham, and Hoskin 1994; Van der Linden et al. 2004). Besides chemotherapy further hormonal manipulation with prednisone or dexamethasone may have some benefit as well. Glucocorticoids may lead to PSA response and/or relief of symptoms in patients with late-stage prostate cancer (Tannock et al. 1989). Some investigators have suggested that the superior results of regimens with taxanes may be due in part to the dexamethasone that is administered to avoid toxic reactions to these drugs. However, most patients have already received substantial treatment with glucocorticoids concurrent with first-line chemotherapy, so their potential benefit in later stages is probably minimal.

Other treatment options in the advanced stage of prostatic cancer include ketoconazole and estrogens. Inhibition of steroid synthesis by ketoconazole may increase the probability of an anti-androgen withdrawal response, although this did not translate into improved survival (Small et al. 2004). Estrogens may improve symptoms but caution must be used because of their ability to stimulate thrombosis and cardiovascular events. Estrogens were found to be equivalent to estramustine (which contains estrogen), probably as its activity is largely due to the estrogen component (Small et al. 2000). Transdermal administration of oestrogens through a patch avoids the entero-hepatic circulation and therefore it should not be associated with the same level of cardiovascular toxicity. Early data confirm the safety and efficacy of oestrogen patches as hormonal treatment in prostate cancer patients (Langley et al. 2008).

Currently new non chemotherapeutic options are studied such as endotheline antagonists (James et al. 2008) and abiraterone acetate, a potent, selective, small-molecule inhibitor of cytochrome P (CYP) 17, a key enzyme in androgen synthesis (Attard et al. 2008). Many other agents are being developed (Tu and Lin 2008).

\section{Bone seeking radiopharmaceuticals}

Bone seeking radiopharmaceuticals have proven to be useful for treatment of more generalized bone pain. All patients will finally progress to end stage disease with multiple skeletal metastases. These patients may receive bone seeking radiopharmaceuticals for generalized painful disease (Berthold, Sternberg, and Tannock 2005). The association of integrated cancer centres in the Netherlands (VIKC) developed an evidence based guideline on the diagnosis and treatment of pain in cancer patients. Radionuclide treatment of cancer patients with metastatic bone pain (so called bone seeking radiopharmaceuticals) was evaluated using all available literature (VIKC 2008). The conclusions are stated together with their level of evidence in Table 1.

Most of the patients who have participated in the mentioned trials were heavily pre-treated patients with previous radiotherapy, chemotherapy and/or hormone therapy. It was recommended that radionuclide treatment with bone seeking radiopharmaceuticals is indicated in patients with multifocal pain originating from osteoblastic skeletal metastases. 
Repeated treatments are indicated after an initial response to treatment. The committee had the opinion that combined multimodality treatment should be performed in a trial setting. Further research in that field is warranted (VIKC 2008).

Radionuclide therapy with bone seeking radiopharmaceutical agents has been long used. It evolved from agents like ${ }^{32} \mathrm{P}$-phosphate to newer agents like ${ }^{188} \mathrm{Re}-\mathrm{HEDP}$ or ${ }^{223} \mathrm{Ra}$ (Table 2). Bone seeking radiopharmaceuticals consist of a radionuclide for the therapeutic effect and a carrier to reach the target site at the bone matrix level. Sometimes the carrier and the radionuclide are one and the same. This is the case for ${ }^{32} \mathrm{P}$-phosphate, ${ }^{223} \mathrm{Ra}$ and ${ }^{89} \mathrm{Sr}$. These radiopharmaceuticals behave as physiologic phosphate (32P-phosphate) or $\mathrm{Ca}^{2+}$-analogues (223 $\mathrm{Ra}$ and ${ }^{89} \mathrm{Sr}$ ). They do not need a non-radioactive substance as a carrier to reach the target. Carriers like hydroxyethylenediphosphonic acid (HEDP in 186Re-HEDP) and ethylenediaminetetramethylenephosphonic acid (EDTMP in ${ }^{153} \mathrm{Sm}$-EDTMP) are being used in other bone seeking radiopharmaceuticals. They behave as bisphosphonates. These differences influence the biodistribution and pharmacokinetics of the pharmaceutical. Other differences between these agents include the radiation type, the radiation energy and the radionuclide half-life.

One thing that never changed during the last decades and stimulated the search for new agents was the conflict between efficacy and toxicity. The latter consisting of bone marrow suppression in particular. This has even led to a change of indication for the use of ${ }^{32} \mathrm{P}-$ phosphate. It is not used anymore for the palliation of metastatic bone pain but instead for the treatment of myeloproliferative diseases, making use of its bone marrow suppressive potential (Berlin 2000; Cheung and Driedger 1980). Fortunately newer agents have proved to be feasible and relatively safe for the palliative treatment of osseous metastases with acceptable and reversible bone marrow toxicity.

\begin{tabular}{lll}
\hline \multicolumn{1}{c}{ Conclusion } & Evidence a & \multicolumn{1}{c}{ Study type b } \\
\hline It has been proven that treatment with & A1 (Bauman et al. 2005; Finlay, \\
bone seeking radiopharmaceuticals & Mason, and Shelley 2005; \\
yields a better pain response than & McQuay et al. 2008; Roque i \\
treatment with placebo in patients with & Level 1 & Figuls et al. 2008) \\
painful osseous metastases from diverse & & A2 (Han et al. 2002; Lewington et \\
cancers including prostate, breast and & al. 1991; Maxon, III et al. 1991; \\
lung cancer. & Sartor et al. 2004; Serafini et al. \\
& 1998)
\end{tabular}

It has been proven that no difference exists with regard to local pain response between treatment with ${ }^{89} \mathrm{Sr}$-Chloride or external beam radiotherapy in patients Level 1 A2 (Oosterhof et al. 2003; Quilty with painful osseous metastases from a prostate carcinoma.

It is likely that no difference exists with regard to pain response between treatment with ${ }^{89} \mathrm{Sr}$-Chloride and ${ }^{186} \mathrm{Re}-$ HEDP in patients with painful osseous Level 2 A2 (Sciuto et al. 2001) metastases. 


\begin{tabular}{ccc}
\hline Conclusion & Evidence $^{\mathrm{a}}$ & Study type $^{\mathrm{b}}$ \\
\hline
\end{tabular}

It is likely that the onset of the pain response of ${ }^{186} \mathrm{Re}-\mathrm{HEDP}$ is faster than the onset of the pain response of ${ }^{89} \mathrm{Sr}-$ Chloride in patients with painful osseous metastases from a breast carcinoma.

It is likely that combined treatment with ${ }^{89} \mathrm{Sr}$-Chloride and chemotherapy (platinum based) yields a better pain response than treatment without chemotherapy in patients with painful osseous metastases from a prostate carcinoma.

It has been suggested that adding ${ }^{89} \mathrm{Sr}$ Chloride to chemotherapy may lead to improved survival and a longer duration of the pain response compared to treatment with chemotherapy alone.

It has been suggested that no difference exists with regard to the pain response after treatment with chemotherapy or ${ }^{89} \mathrm{Sr}-\mathrm{Ch}$ loride in patients with painful osseous metastases from a prostate carcinoma.

No conclusions can be drawn on the value of adding ${ }^{89} \mathrm{Sr}$-Chloride to external beam radiotherapy in patients with painful osseous metastases from a prostate carcinoma because of conflicting results.
Level 2 A2 (Sciuto et al. 2001)

Level 2 A2 (Sciuto et al. 2002)

C (Sciuto et al. 1996)
Level 3 B (Tu et al. 2001)

Level 3 B (Nilsson et al. 2005)

a Level of evidence: 1) A1 or at least two independent and consistent A2 studies; 2) One A2 study or at least two independent and consistent B studies; 3) One B or C study; 4) Professional opinion.

${ }^{b}$ Quality and methodology of studies: A1) Systemic review of at least two independent A2 trials; A2)

Double-blind randomized trial of sufficient size and quality (comparison with a reference test ('gold standard'), defined endpoints, independent evaluation of both tests, no confounding); B) Comparative trial not meeting A2 criteria;

c Non-comparative trial; D) Professional opinion.

Table 1. Evidence based conclusions on treatment with bone seeking radiopharmaceuticals (VIKC 2008). 


\begin{tabular}{|c|c|c|c|}
\hline Radiopharmaceutical & Half-life (days) & $\begin{array}{c}\beta \text {-emission } \mathrm{MeV} \\
\max (\text { mean) }\end{array}$ & $\begin{array}{c}\gamma \text {-emission } \\
\operatorname{keV}(\%)\end{array}$ \\
\hline 188Re-HEDP & 0.7 & $2.12(0.76)$ & $155(15 \%)$ \\
\hline 153Sm-EDTMP & 1.93 & $0.81(0.23)$ & $103(29 \%)$ \\
\hline 186Re-HEDP & 3.7 & $1.07(0.35)$ & $137(9 \%)$ \\
\hline 177Lu-EDTMP & 6.7 & $0.497(0.15)$ & $208(11 \%)$ \\
\hline 223Ra & 11.4 & \multicolumn{2}{|c|}{ Emits alfa-particles of circa $5.7 \mathrm{MeV}$} \\
\hline 117mSn-DTPA & 13.6 & \multicolumn{2}{|c|}{ Emits conversion electrons $127-152 \mathrm{keV}$} \\
\hline 32P-Phosphate & 14.3 & $1.71(0.70)$ & None \\
\hline${ }^{89} \mathrm{Sr}$ & 50.5 & $1.46(0.58)$ & $910(0.01 \%)$ \\
\hline
\end{tabular}

Table 2. Bone seeking radiopharmaceuticals categorized by half-life.

All patients with proven osteoblastic (or mixed type) skeletal metastases that accumulate 99mTc-HDP on skeletal scintigraphy may be candidates for treatment with bone seeking radiopharmaceuticals. They may be cancer patients with advanced disease originating from prostate cancer, breast cancer, lung cancer, medullary thyroid carcinoma, or other tumors (i.e. bronchial carcinoid tumors, medulloblastoma). In routine clinical practice the vast majority of patients are prostate cancer patients. In these patients the incidence of skeletal metastases is very high. They cause high morbidity and mortality (DePuy et al. 2007; Saarto et al. 2002). Metastases originating from prostate cancer are pure osteoblastic with relatively high radionuclide uptake, resulting in high tumor to non-tumor ratio's. And last but not least other treatment options are limited in advanced stages of this disease.

In the growing field of radionuclide therapy many new radiopharmaceuticals are being developed. At the moment ${ }^{89} \mathrm{SrCl}_{2}$ (Metastron ${ }^{\circledR}$ ) and ${ }^{153 \mathrm{Sm}-E D T M P}$ (Quadramet ${ }^{\circledR}$ ) are both FDA approved. Together with 186Re-HEDP (registered in some countries, not in the Netherlands) these bone seeking radiopharmaceuticals are mostly used today.

\section{Bone seeking radiopharmaceuticals in clinical practice}

\subsection{Radiation safety considerations}

Patients treated with any kind of radionuclide treatment must be regarded as a potential risk for public health because of a potential radiation hazard. Good understanding of the radionuclide used, its physical characteristics, its biodistribution and its pharmacokinetics, will allow us to draw proper guidelines for this kind of treatment. Does the patient need to be confined after treatment? Are we able to identify the radiation hazard from a qualitative and quantitative perspective? What does that mean for an individual patient in relation to its environment?

Patients treated with $89 \mathrm{SrCl} 2,186 \mathrm{Re}-\mathrm{HEDP}$ or $153 \mathrm{Sm}$-EDTMP are a source of radiation, including beta-radiation that has proven to be measurable outside the patient. Betaparticles in superficial tissue (such as in bones, blood vessels) cross the skin and contribute to the ambient equivalent dose. This aspect must be considered when using beta-emitting radiopharmaceuticals in general. The calculated effective doses for bystanders are well below the recommended values and do not lead to unacceptable additional radiation burden to health care workers and patients' families. The mean total effective doses absorbed by bystanders at $30 \mathrm{~cm}$ distance from a patient are approximately $0.02 \mathrm{mSv}$ for $89 \mathrm{SrCl} 2,0.3 \mathrm{mSv}$ for 186Re-HEDP, and $1.6 \mathrm{mSv}$ for 153Sm-EDTMP (Lam et al. 2009b). These observations however should be placed in some perspective. First the 
individual variation in effective dose to bystanders and second the potential risk of internal contamination of bystanders.

The total effective dose, as given above, is estimated for bystanders who reside at exactly 30 $\mathrm{cm}$ from the patient for an indefinite time. Because this is never the case, these estimations must be corrected for variations in time and distance between bystanders and patients. In a Dutch Ministry of Housing, Spatial Planning and Environment publication accurate calculations were made to cover the various persons who may have contact with patients (VRO92; VROM 2005). These calculations are based on residence times $\mathrm{T}$ (in fractions of days) with the patient and distances $R$ (in meters) from the patient. The actual effective doses for bystanders will depend on residence times and distances in relation to the patient. Estimations were made for residence times and distances during a 24-hour period. This was done in the case of an elderly patient in relation to his or her partner and in the case of a patient in relation to his or her child (Tables 3 and 4).

\begin{tabular}{lccc|ccc}
\hline & \multicolumn{3}{c|}{ Without instruction } & \multicolumn{3}{c}{ With instruction } \\
\cline { 2 - 7 } & $\begin{array}{c}\text { Residence } \\
\text { time } \\
(\mathrm{hrs} / 24 \mathrm{hrs})\end{array}$ & $\begin{array}{c}\text { Distance } \\
(\mathrm{m})\end{array}$ & $\begin{array}{c}\text { Correction } \\
\text { factor a }\end{array}$ & $\begin{array}{c}\text { Residence } \\
\text { time } \\
\text { (hrs/24hrs })\end{array}$ & $\begin{array}{c}\text { Distance } \\
(\mathrm{m})\end{array}$ & $\begin{array}{c}\text { Correction } \\
\text { factor a }\end{array}$ \\
\hline $\begin{array}{l}\text { outdoors } \\
\text { activities }\end{array}$ & $3 / 24$ & - & 0 & $3 / 24$ & - & 0 \\
watching & $5 / 24$ & 0.5 & 0.075 & $5 / 24$ & 2 & 0.0047 \\
TV & $2 / 24$ & 1 & 0.0075 & $2 / 24$ & 1 & 0.0075 \\
dinner & $8 / 24$ & 0.7 & 0.061 & $8 / 24$ & 2 & 0.0075 \\
sleeping & $6 / 24$ & 3 & 0.0025 & $6 / 24$ & 3 & 0.0025 \\
other \\
total
\end{tabular}

a Correction factor applicable for measurements at $30 \mathrm{~cm}$ from the patient using the inverse-square law

Table 3. Effective dose (external radiation) of an elderly partner with and without instructions.

Assuming that the estimated distances and times are a reflection of reality, corrections were made for these circumstances. Estimations of the effective doses for these persons (partner and child) are given for the three most used radiopharmaceuticals. In the case of a patient and his or her partner, without instructions a correction factor of $0.15(15 \%)$ was applied. This is explained by the fact that bystanders do not stay within $30 \mathrm{~cm}$ of patients 24 hours a day. Because of work and other activities a correction factor should be applied. As an example a correction factor of 0.15 may be applied. The effective dose may be further reduced by instructing the patients and their families to keep distance as much as reasonably possible (e.g. watching TV and sleeping apart). With proper instructions to 
family, residence times may be reduced and distances increased, lowering the correction factor to as low as $0.02(2 \%)$, an almost eight-fold decrease in radiation burden to bystanders (Table 3). In the case of a patient and his or her child an estimated correction factor of about $0.43(43 \%)$ should be applied without instructions and $0.11(11 \%)$ with instructions. In all instances effective doses will be $<1 \mathrm{mSv}$ and with proper instructions they will be $<0.1 \mathrm{mSv}$ or even $<0.01 \mathrm{mSv}$ (Tables 3 and 4). It may be concluded that patients treated with bone seeking radiopharmaceuticals do not pose any threat to others.

\begin{tabular}{|c|c|c|c|c|c|c|}
\hline & \multicolumn{3}{|c|}{ Without instruction } & \multicolumn{3}{|c|}{ With instruction } \\
\hline & $\begin{array}{c}\text { Residence } \\
\text { time } \\
\text { (hrs/24hrs) }\end{array}$ & $\begin{array}{c}\text { Distance } \\
(\mathrm{m})\end{array}$ & $\begin{array}{c}\text { Correction } \\
\text { factor a }\end{array}$ & $\begin{array}{l}\text { Residence } \\
\text { time } \\
\text { (hrs/24hrs) }\end{array}$ & $\begin{array}{c}\text { Distance } \\
(\mathrm{m})\end{array}$ & $\begin{array}{c}\text { Correction } \\
\text { factor a }\end{array}$ \\
\hline \multirow{7}{*}{$\begin{array}{l}\text { playing } \\
\text { close } \\
\text { contact } \\
\text { dinner } \\
\text { sleeping } \\
\text { other } \\
\text { total } \\
\end{array}$} & $8 / 24$ & 4 & 0.0019 & $8 / 24$ & 4 & 0.0019 \\
\hline & $1 / 24$ & 0.1 & 0.375 & $0.25 / 24$ & 0.1 & 0.094 \\
\hline & $2 / 24$ & 0.5 & 0.03 & $2 / 24$ & 2 & 0.0019 \\
\hline & $10 / 24$ & - & - & $10 / 24$ & - & - \\
\hline & $3 / 24$ & 2 & 0.0094 & $3 / 24$ & 2 & 0.0094 \\
\hline & & & 0.43 & & & 0.11 \\
\hline & \multicolumn{3}{|c|}{ Effective dose (mSv) } & \multicolumn{3}{|c|}{ Effective dose (mSv) } \\
\hline $\begin{array}{l}\text { EDTMP- } \\
\text { EDTMP }\end{array}$ & \multicolumn{3}{|c|}{0.69} & \multicolumn{3}{|c|}{0.18} \\
\hline $\begin{array}{l}\text { 186Re- } \\
\text { HEDP }\end{array}$ & \multicolumn{3}{|c|}{0.13} & \multicolumn{3}{|c|}{0.03} \\
\hline $\begin{array}{l}{ }^{89} \mathrm{Sr}- \\
\text { Chloride }\end{array}$ & \multicolumn{3}{|c|}{$<0.01$} & \multicolumn{3}{|c|}{$<0.01$} \\
\hline
\end{tabular}

a Correction factor applicable for measurements at $30 \mathrm{~cm}$ from the patient using the inversesquare law

Table 4. Effective dose (external radiation) of a young child with and without instructions.

However, an exception has to be made considering urinary excretion of activity and the possible internal contamination of bystanders. Besides radiation exposure to non-patients from direct emission by the patient, another potential radiation hazard is formed by excreted radioactivity. The calculated mean total urinary excretion percentage of ${ }^{89} \mathrm{Sr}$ during the first 3 days after administration was 16\% (Lam et al. 2009b). Using a hypothetical contamination scenario, that is used in radiation protection evaluation (VROM 2005), that $0.01 \%$ of the excreted amount of radioactivity will cause internal contamination to nonpatients closely related to the patient, an internal dosage of $0.0024 \mathrm{MBq}$ for ${ }^{89} \mathrm{SrCl}_{2}$ therapy (administered dose of $150 \mathrm{MBq}$ ) was calculated. For ${ }^{186}$ Re-HEDP therapy (administered dose of $1295 \mathrm{MBq})$, the corresponding amount of radioactivity will be $0.064 \mathrm{MBq}(49 \%$ of the injected dose is excreted (de Klerk et al. 1992)). After treatment with $37 \mathrm{MBq} / \mathrm{kg} 153 \mathrm{Sm}-$ EDTMP $53.1 \% \pm 15.1 \%$ of the administered dose was excreted in urine during the first 48 hours (Lam et al. 2007). Potential contamination with $0.01 \%$ of the excreted radioactivity will lead to an internal dosage of $0.15 \mathrm{MBq}{ }^{153} \mathrm{Sm}$-EDTMP. The dose conversion coefficient for ingestion of ${ }^{89} \mathrm{Sr}$ is $\left(\mathrm{e}_{\mathrm{ing}}\right)=2.6 \times 10^{-9} \mathrm{~Sv} / \mathrm{Bq}$, of ${ }^{186} \mathrm{Re}\left(\mathrm{e}_{\mathrm{ing}}\right)=1.510^{-9} \mathrm{~Sv} / \mathrm{Bq}$, and of ${ }^{153} \mathrm{Sm}\left(\mathrm{e}_{\text {ing }}\right)$ $=7.410^{-10} \mathrm{~Sv} / \mathrm{Bq}$. The present data show that the effective radiation absorbed dose, caused 
by a potential internal contamination ( $0.01 \%$ of the administered dose), is 6.2 microSv for

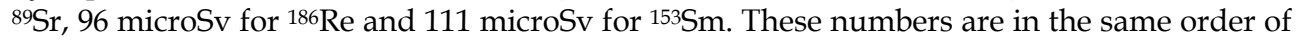
magnitude as the numbers given for external exposure (Table 3 and 4).

The total effective dose for non-patients may be caused by both external radiation exposure and internal contamination. In contrast to the mean effective dose caused by external radiation, the effective dose after ingestion of $0.01 \%$ of the administered dose is hypothetical and may be much higher or much lower. In the case of ${ }^{131} \mathrm{I}$ it proved to be less. The uptake of ${ }_{131}$ I in the thyroid of family members was measured (Buchan and Brindle 1970). A maximum uptake of $3.8 \mathrm{~Bq}$ per $\mathrm{MBq}$ administered was found. So on one hand it must be considered that $0.01 \%$ is a hypothetical figure, while the external radiation exposure is a fact. On the other hand, internal contamination poses a real threat to non-patients. Patients that were treated with bone-seeking radiopharmaceuticals are often severely disabled (in contrast to ${ }^{131}$ I patients). Especially in the case of prostate cancer patients, they often have dysurea. Personal hygiene is not as obvious as it is to others. It is therefore advisable to give the patients simple, easy-to-follow instructions, in order to reduce the risk for non-patients. Using a separate toilet, sitting while urinating and washing hands afterwards, are highly recommended. In the case of incontinence, patients must be catheterized for a certain time depending on urinary excretion of the administered activity. Due to fast renal excretion this may be 12 hours after injection of ${ }^{186} \mathrm{Re}-\mathrm{HEDP}$ and ${ }^{153 \mathrm{Sm}-\mathrm{EDTMP} .}{ }^{89} \mathrm{SrCl}_{2}$ is being administered in relatively low doses and therefore has a relatively low risk for high effective dose due to ingestion of this radiopharmaceutical (6.2 microSv for ${ }^{89} \mathrm{Sr}$ ). These patients do not have to be catheterized. The risk for significant internal contamination of non-patients is much lower and acceptable for this radiopharmaceutical.

In general it is advised to hospitalize patients treated with 186Re-HEDP and 153Sm-EDTMP for at least 8 hours. This is mostly based on urinary excretion and the risk for internal contamination, because the radiation exposure to non-patients is $<20$ microSv/hour (1 meter from the patients) directly or within a few hours after administration in all cases. In the case of incontinence it is advised to treat patients with either ${ }^{186} \mathrm{Re}-\mathrm{HEDP}$ or ${ }^{153 \mathrm{Sm}}$ EDTMP with a urinary catheter for 12 hours after administration. Patients treated with ${ }^{89} \mathrm{SrCl}_{2}$ may return home directly.

After discharge it is advisable to keep distance where possible (Table 3 and 4), following the ALARA ('as low as reasonably achievable') principles. This means, for example, that older patients (> 60 years) may still sleep close to their older partner, while being more stringent towards younger relatives to avoid any unnecessary radiation dose. The ICRP has proposed an effective dose limit of $1 \mathrm{mSv}$ per year for individuals. In special circumstances a higher value may be allowed in a single year provided that the average over 5 years does not exceed $1 \mathrm{mSv}$ per year. In clinical practice, the use of bone-seeking radiopharmaceuticals will give rise to a degree of radiation exposure to all those in contact with patients, albeit in very low doses. The present results further confirm the safety of treatment with boneseeking radiopharmaceuticals.

\subsection{Treatment recommendations}

${ }^{89} \mathrm{SrCl}_{2}\left(\mathrm{Metastron}^{\circledR}\right)$ and ${ }^{153 \mathrm{Sm}-\mathrm{EDTMP}}$ (Quadramet ${ }^{\circledR}$ ) are both FDA approved and registered in the Netherlands. Together with ${ }^{186}$ Re-HEDP (registered in some countries, not in the Netherlands) these bone seeking radiopharmaceuticals are mostly used. Most of the randomized double-blind placebo controlled trials have been performed using ${ }^{89} \mathrm{SrCl}_{2}$ or ${ }^{153 \mathrm{Sm}}$ - 
EDTMP. An evidence based approach would be a choice between these radiopharmaceuticals. One of the differences between these two is the magnitude and rate of renal excretion. Both may be used without confining a patient to the hospital but from a radiation safety perspective it is advised to keep the patient in a controlled setting for at least 8 hours after injection in the

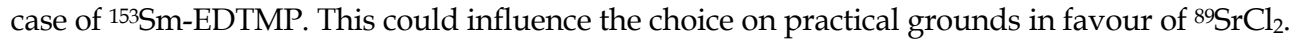
Not all nuclear medicine departments have such facilities.

Other differences include the longer half-life and higher energy (with higher range in tissue)

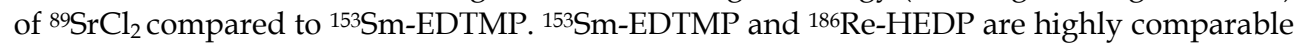
with regard to energy and half-life. Most comparative randomized studies have been performed using ${ }^{89} \mathrm{SrCl}_{2}$ and ${ }^{186} \mathrm{Re}-\mathrm{HEDP}$. It was found that no difference exist with regard to pain response between treatment with ${ }^{89} \mathrm{Sr}$-Chloride and ${ }^{186} \mathrm{Re}-\mathrm{HEDP}$ in patients with painful osseous metastases (Piffanelli et al. 2001; Sciuto et al. 2001). And that the onset of the pain response of ${ }^{186} \mathrm{Re}-\mathrm{HEDP}$ is faster than the onset of the pain response of ${ }^{89} \mathrm{Sr}$-Chloride in patients with painful osseous metastases from a breast carcinoma (Sciuto et al. 2001). So one might argue that when a faster pain response is indicated one should use 186 Re-HEDP or

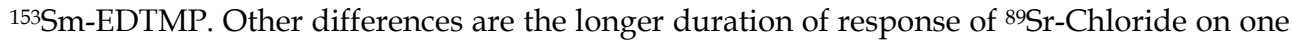
hand, and the prolonged bone marrow toxicity of ${ }^{89} \mathrm{Sr}$-Chloride on the other hand. These differences were not investigated in direct comparative studies but should be considered nevertheless. In summary: in favour of ${ }^{89} \mathrm{Sr}$-Chloride are:

- No confinement necessary

- Longer duration of response (suitable in relatively good clinical condition in which a prompt response is not warranted)

In favour of ${ }^{186} \mathrm{Re}-\mathrm{HEDP}$ or ${ }^{153 \mathrm{Sm}-\mathrm{EDTMP} \text { are: }}$

- Fast response (suitable in bad clinical condition in which immediate response is wanted)

- Favourable toxicity profile (suitable in heavily pre-treated patients, in wide spread metastatic disease and possibly in combination with other myelotoxic treatments)

In most cases today a fast response is needed. Besides that most patients, including prostate cancer patients, are heavily pre-treated. They have end stage disease with minimal bone marrow reserve. And last but not least short-living bone seeking radiopharmaceuticals like 186 Re-HEDP, $153 \mathrm{Sm}$-EDTMP and others may prove to be more suitable in combination with other treatment modalities, not just because of their toxicity profile but also because of their high dose rate, offering an effective treatment with fast recovery.

\section{Enhancement of efficacy}

A major concern in the treatment of prostate cancer patients in the more advanced stage of the disease is the delicate balance between efficacy and toxicity. Treatment of metastatic bone pain with analgesics or localized external beam radiotherapy is relatively safe and easy. Treatment with bone seeking radiopharmaceuticals may be more appropriate in selected cases but efficacy is sometimes disappointing and bone marrow toxicity may be high in individual patients. Enhancement of overall efficacy without increasing toxicity could push the clinical decision algorithm in a positive direction with regard to the use of bone seeking radiopharmaceuticals.

One way of improving overall efficacy is combined treatment. Combined treatment regimens may deliver the beneficial effect of two different treatment modalities. These 
combinations may not only be additive but possibly synergistic to each other, leading to enhancement of overall efficacy with acceptable toxicity.

\subsection{Multimodality treatment}

The propensity of prostate cancer to metastasize to bone and the prognostic significance of bone metastases suggest that effective treatment of bone metastases may provide clinical benefits (DePuy et al. 2007; Sabbatini et al. 1999). With regard to the 'seed' and 'soil' theory on bone metastases the seed may comprise the so-called cancer stem cells. Whereas the soil may comprise a unique microenvironment, that facilitate the growth and survival of cancer stem cells. Targeting the microenvironment may offer another way to improve treatment of prostate cancer bone metastases. The microenvironment consists of osteoclasts, osteoblasts, endothelium and stroma. In the presence of cancer stem cells they interact leading to a disruption in normal coupling between osteoclasts and osteoblasts. An improved understanding of this process will influence how we select agents to target bone metastases and how we design strategies to treat prostate cancer bone metastases. Treatments may be directed to the cancer stem cells, the osteoblasts, the osteoclasts, the endothelium or the stroma (Tu and Lin 2008).

Osteoblasts may be targeted by several pharmaceuticals including bone seeking radiopharmaceuticals. Other osteoblast directed treatments include endothelin-1antagonists (atrasentan) (Carducci et al. 2003), vitamin D analogs (1,25-hydroxyvitamin $\mathrm{D}_{3}$ ) (Beer et al. 2007), monoclonal IGF-1R (insulin-like growth factor-1-receptor) antibodies (Boyle et al. 2001) and CXRC4 (G-protein-coupled receptor) inhibitors (MSX-122), which inhibit the homing behaviour of cancer stem cells. Osteoclast activity may be inhibited by bisphosphonates (zolendronate) (Saad et al. 2002), RANK ligand inhibitors (denosumab) (Lewiecki 2006), tyrosine kinase inhibitors (dasatinib) or IL-6 antagonists (CNTO328) (Nam et al. 2005). And the endothelium and/or stroma may be targeted by anti-angiogenesis therapies. These include the vascular endothelial growth factor (VEGF) receptor antagonists bevacizumab, thalidomide and lenalidomide (Dahut et al. 2004). They reduce VEGF levels and basic fibroblast growth factor, inhibit growth and survival of tumor cells by modulation of adhesion molecules and mediate various cytokines. Also targeted to the endothelium are platelet-derived growth factor receptor (PDGFR) tyrosine kinase inhibitors (imatinib, sunitinib and tandutinib) (Ko et al. 2001). They may also have anti-angiogenetic potential. Most of these agents are under investigation. Many clinical studies in prostate cancer patients are ongoing (Tu and Lin 2008).

Combining different treatment modalities may be interesting because of additive effects or synergy effects. In the case of bisphosphonates and bone seeking radiopharmaceuticals the combined use has always been contra-indicated because of presumed interaction at the bone matrix level. It was shown that the combined use of $153 \mathrm{Sm}$-EDTMP and bisphosphonates in patients with hormone-refractory prostate carcinoma is feasible. The combined treatment regimen is safe and may prove to be an effective long-term treatment regimen (Lam et al. 2007).

Another combined treatment regimen would be a combination of bone seeking radiopharmaceuticals and chemotherapy. It is likely that combined treatment with ${ }^{89} \mathrm{Sr}-$ Chloride and chemotherapy (platinum based) yields a better pain response than treatment without chemotherapy in patients with painful osseous metastases from a prostate carcinoma (Sciuto et al. 2002), (Sciuto et al. 1996). And it has been suggested that adding 
${ }^{89}$ Sr-Chloride to chemotherapy may lead to improved survival and a longer duration of the pain response compared to treatment with chemotherapy alone (Tu et al. 2001). Studies are yet limited but they are encouraging. Most of them have been performed using ${ }^{89} \mathrm{Sr}-$ Chloride and some using ${ }^{153 \mathrm{Sm}-E D T M P}$ (Ricci et al. 2007). Patients may possibly have an improved pain response and longer survival. In prostate cancer patients a most interesting choice would be combining docetaxel and a bone seeking radiopharmaceutical. These studies, using ${ }^{153 \mathrm{Sm}-\mathrm{EDTMP}}$ or ${ }^{186} \mathrm{Re}-\mathrm{HEDP}$, are underway. Our group investigated the combination of ${ }^{188}$ Re-HEDP and capecitabine (Xeloda ${ }^{\circledR}$ ) (Lam et al. 2009a). This treatment regimen proved to be feasible and safe. Phase II efficacy testing using the maximum tolerable dose of $2500 \mathrm{mg} / \mathrm{m}^{2} /$ day capecitabine is underway. Capecitabine is primarily used as a radiation sensitizer. It offers a convenient therapy with acceptable toxicity, ease of use as oral tablets and low costs. The same is true for ${ }^{188}$ Re-HEDP. It is homemade on demand and has favourable physical characteristics with a short half-life (16.9 hours) and high betaenergy $\left(\mathrm{E}_{\beta \max } 2.12 \mathrm{MeV} ; \mathrm{E}_{\beta}\right.$ mean $\left.0.76 \mathrm{MeV}\right)$. In theory, this high dose rate could lead to improved efficacy with rapid recovery. This is ideal for combined treatment regimens and for repeated treatment, which has already shown to be favourable with regard to pain response and survival (Palmedo et al. 2003).

Several other combinations using bone seeking radiopharmaceuticals are under investigation. Currently, a randomized phase III study (MDA-3410/CTSU in M.D. Anderson Cancer Center, Houston, Texas) combining weekly doxorubicin $\left(20 \mathrm{mg} / \mathrm{m}^{2}\right)$ with ${ }^{89} \mathrm{Sr}$-Chloride after response to induction chemotherapy is underway, as well as another phase I trial combining docetaxel/prednisone and 153Sm-EDTMP, also at M.D. Anderson ( $\mathrm{Tu}$ and Lin 2008). It remains to be established whether targeting both the tumor (chemotherapy) and bone compartments will improve therapeutic efficacy. This concept is also being tested in multiple other trials, mostly combining docetaxel/prednisone with bone environment directed treatments, like the agents described above ( $\mathrm{Tu}$ and $\mathrm{Lin} 2008$ ). The described studies add to this search for optimized treatment regimens in hormonerefractory prostate cancer patients.

\subsection{Predictions of efficacy and toxicity}

Most patients with advanced prostate cancer have disease limited to the bone, which is notoriously difficult to assess for response, with a small subset having soft tissue lesions. To limit response evaluation to only patients with bidimensionally measurable disease would eliminate $70 \%$ to $80 \%$ of patients who would otherwise be evaluable (Figg et al. 1996). With regard to prostate cancer patients efficacy of treatment has been monitored by PSA in a majority of studies. However it is doubtful whether PSA changes correlate with clinical benefit (Bubley et al. 1999). A 50\% decrease in PSA level seems a reasonable predictor of a favourable outcome, but this was certainly not the case in all studies (Bauer et al. 1999; Sridhara et al. 1995). It was advised not to use PSA level drops as a surrogate marker for survival (Bubley et al. 1999).

In patients with advanced disease, survival and quality of life, including pain palliation, are the most important criteria of clinical response. Besides survival these parameters are difficult to measure and subject to errors. With an attempt to standardize treatment response monitoring in cancer patients the European Organization for Research and Treatment of Cancer (EORTC) has developed quality of life questionnaires (EORTC QLQC30 version 3.0). They were validated in many clinical trials (Aaronson et al. 1993). They 
include questions on global health status / quality of life, functional scales and symptom scales. The functional scales are subdivided in physical functioning, role functioning, emotional functioning, cognitive functioning and social functioning. The symptom scales are subdivided in fatigue, nausea and vomiting, pain, dyspnoea, insomnia, appetite loss, constipation, diarrhoea and financial difficulties. Each domain was scored on a scale of 0 100, according to the EORTC scoring manual (Fayers et al. 2001). The changes in scores may be used to monitor clinical effect.

Other often used clinical monitoring tools include the visual analogue scales (VAS) to monitor pain, changes in analgesic intake and, more basic, the physician's assessment based on anamnesis and physical examination. A matter of debate is the frequency of evaluation. It seems that daily assessment is necessary to appreciate the wide variation in clinical status that patients may experience from day to day (Han et al. 2002). In all cases it is difficult to find a good balance between accuracy and compliance. PSA is therefore still popular to measure an objective level of response (Bubley et al. 1999; Scher et al. 2008).

However, other predictors of response may prove to be much more reliable than PSA. In hormone-refractory prostate cancer patients in advanced stages of their disease the extent of metastatic disease in the skeleton is of high prognostic significance (Sabbatini et al. 1999). Furthermore it was shown that patients with advanced disease who had experienced a socalled Skeletal Related Event (defined as: pathologic fracture, spinal cord compression with vertebral compression fracture, the need for surgery to treat or prevent pathologic fractures or spinal cord compression, or the need for radiation to bone) had a significantly worse survival and poorer quality of life, in comparison with patients who had not experienced a Skeletal Related Event (DePuy et al. 2007). These results further confirm the importance to treat skeletal metastases adequately. Markers of skeletal metabolism were found to be related to outcome and survival. In several clinical studies in prostate cancer patients it was observed that markers of bone metabolism were able to predict outcome, both as absolute levels pretreatment and as changes after treatment (Jung et al. 2004; Lein et al. 2007). In large series of cancer patients, including a majority of prostate cancer patients, treated with zoledronic acid, it was found that baseline levels of the bone marker urinary $\mathrm{N}$-terminal type I collagen peptide [NTX], as well as changes of NTX after treatment were able to predict improved survival (Coleman et al. 2005; Lipton et al. 2008). Other studies emphasized the importance of the bone marker serum bone specific alkaline phosphatase [BAP] as a predictor of outcome (Cook et al. 2006; Smith et al. 2007). Most of these studies used the same bone markers as we did in the study on the combined treatment of prostate cancer patients with 153Sm-EDTMP and zoledronic acid (i.e. NTX, BAP and serum procollagen type I N propeptide [PINP],). Although this study comprised a small patient population the results are of interest because they confirm the utility of these markers as predictors of clinical outcome, even in small numbers (Lam et al. 2007). Besides, they were first tested in the treatment monitoring of bone seeking radiopharmaceuticals. The bone formation markers BAP and PINP were in agreement with the clinical effect of the combined treatment regimen evaluated by EORTC questionnaires. The bone resorption marker NTX and PSA were not in agreement with the clinical effect. This supports the hypothesis that the extent of osteoblastic metastasis in hormone-refractory prostate cancer patients is an important parameter for clinical outcome. Both treatment itself and treatment monitoring should be directed to these osteoblastic metastases. Bone markers may well prove to be very useful predictors of clinical effect in the treatment with bone seeking radiopharmaceuticals. They should be used in future trials. 
Last but not least the importance of imaging modalities should be mentioned with regard to individualized treatment monitoring. Functional rather than anatomical imaging techniques may be used to predict response. Several PET (Positron Emission Tomography) techniques are being developed for this purpose (John et al. 2008; Price et al. 2002). In fact, functional imaging will prove to be one of the major contributions of nuclear medicine to clinical oncology in the future. Does the treatment work? That question needs to be answered for each oncologic treatment on an individual basis. Nuclear imaging and PET in particular may be helpful.

Besides predictors of efficacy, predictors of toxicity are equally important for individualized patient management. Radiopharmaceuticals are important resources in the management of bone pain, but they need to be utilized in a manner that does not prevent other systemic therapy (Rago 1998). Thrombocytopenia is the dose limiting factor in treatment of painful bone metastases with bone seeking radiopharmaceuticals. De Klerk et al evaluated thrombocytopenia in patients with hormone refractory prostate carcinoma, treated with 186Re-HEDP (de Klerk et al. 1994). As an index of the extent of bone involvement, the bone scan index (BSI) was determined from the pre-treatment $99 \mathrm{mTc}-\mathrm{HDP}$ scintigram. The BSI is a tool to describe the extent of skeletal metastases on a scale from 0 to $100 \%$ (Blake et al. 1986). They described a functional relation $(\mathrm{r}=0.78 ; \mathrm{p}<0.001)$ of the percentage of platelet decrease after treatment with the extent and distribution of skeletal metastases (BSI) and administered activity, normalized to standard body surface area. Using this relation, it is possible to predict thrombocytopenia by pre-treatment skeletal scintigraphy and to adjust the dosage for each patient to avoid unacceptable toxicity (de Klerk et al. 1994).

However, more sophisticated indices of bone marrow function might also be of paramount importance. Recently, some very interesting reports have been published on 'reticulated platelets' (Briggs et al. 2004; Wang et al. 2002). In systemic radionuclide therapy, the megakaryocyte seems to be most vulnerable to radiation. It is of great interest to gain more knowledge of bone marrow function pre-treatment using 'biological' parameters like 'reticulated platelets'. These newly released platelets are larger and contain RNA. They were suggested to be the platelet analogue of the red cell reticulocyt. Assessment of platelet production using 'reticulated platelets' would distinguish between thrombocytopenia due to bone marrow failure and impending bone marrow recovery after cytotoxic therapy or thrombocytopenia due to increased peripheral platelet destruction and turnover. In both cases platelet levels are low, but in the latter 'reticulated platelet' levels will be high due to increased production (Wang et al. 2002). This noninvasive measurement could further increase our knowledge of platelet production and the influence of radiation on this process.

By adding hematological, chemical and biological parameters, combined with the bone scan index, body surface area, administered activity and retained activity, an extended version of 'De Klerk's formula' may be developed. This is probably best done as a so called nomogram. Smaletz et al developed a nomogram to predict survival for patients with hormone refractory prostate carcinoma (Smaletz et al. 2002). A nomogram is a model in which individual parameters lead to a chance (from 0 to $100 \%$ ) to experience a predefined outcome. The outcome may be defined as survival or for example toxicity. Such a model can be made to predict hematological toxicity (thrombocytopenia, leucopenia) after treatment with bone seeking radiopharmaceuticals to improve individualized patient management. 


\section{Future prospects}

Several future implications have already been discussed above. One of the most important developments for bone seeking radiopharmaceuticals will be individualized medicine. The search for an optimized balance between efficacy and toxicity will be found rather in an individualized treatment plan than in new agents. Good predictors of efficacy and toxicity are needed for treatment monitoring. Several potential candidates were described above. They may be found in clinical evaluation, imaging and several laboratory parameters. A combination of these parameters may lead to a prediction model which may be used in daily practice.

Multimodality treatment has also been described above. Many combinations have potential. It has to be investigated which regimen will be most effective. Besides a direct anti-tumor effect which may be reached with chemotherapy (i.e. docetaxel), it is recognized that treatment directed to the bony environment may add to the overall efficacy of the treatment regimen. Bone seeking radiopharmaceuticals are bone-directed and may be of value in combination with other treatment modalities. Besides combinations with chemotherapy or bisphosphonates other interesting combinations may include for example atrasentan, or denosumab.

An important other issue is the timing of treatment with bone seeking radiopharmaceuticals. The frequency and interval of sequential treatment should be considered. Bone seeking radiopharmaceuticals are mostly used as a single shot treatment. When patients respond to the treatment repeated treatment is considered. In most instances this does not happen with a planned interval in mind but rather when symptoms reappear after an initial response. In fact this might be too late. It seems better to treat patients sequentially before symptoms reoccur. This was confirmed in a randomized controlled trial in prostate cancer patients treated with two dosages of $1.1 \mathrm{mCi}{ }^{188}$ Re-HEDP with an interval of eight weeks compared to single shot treatment. They did not only find an improved pain response with longer duration but surprisingly an improved survival as well (Palmedo et al. 2003). Safety of repeated treatment with bone seeking radiopharmaceuticals was also confirmed (Sartor et al. 2007). This enhancement of efficacy may be attributed to chronic inhibition of osseous metabolism preventing cancer cells to thrive in the bony environment. Most other oncologic compounds are used in a repeated fashion using several cycles to reach sufficient effect. It should be investigated which multiple treatment regimen is most suitable for bone seeking radiopharmaceuticals, and whether it is safe and more effective. It may be suggested that the best result in hormone-refractory will be reached using a multimodality treatment regimen with fractionation of all treatments to be effective over a prolonged period of time.

\section{Conclusion}

Treatment with bone seeking radiopharmaceuticals in patients with multiple painful skeletal metastases is safe and effective when proper protocols are being used. Important improvements may be found in multimodality treatment in long-term treatment regimens and in individualized patient management. Identification of powerful indicators of toxicity and efficacy may guide patient selection and therapy monitoring to optimize the patient's outcome. This may possibly lead us beyond pain palliation towards improvement of survival. 


\section{References}

Aaronson NK et al (1993) The European Organization for Research and Treatment of Cancer QLQ-C30: a quality-of-life instrument for use in international clinical trials in oncology. J.Natl.Cancer Inst. 85 (5):365-376.

Attard G et al (2008) Phase I clinical trial of a selective inhibitor of CYP17, abiraterone acetate, confirms that castration-resistant prostate cancer commonly remains hormone driven. J.Clin.Oncol. 26 (28):4563-4571.

Auclerc G et al (2000) Management of advanced prostate cancer. Oncologist. 5 (1):36-44.

Bauer KS et al (1999) A pharmacokinetically guided Phase II study of carboxyamido-triazole in androgen-independent prostate cancer. Clin.Cancer Res. 5 (9):2324-2329.

Bauman G et al (2005) Radiopharmaceuticals for the palliation of painful bone metastasis-a systemic review. Radiother.Oncol. 75 (3):258-270.

Beer TM et al (2007) Double-blinded randomized study of high-dose calcitriol plus docetaxel compared with placebo plus docetaxel in androgen-independent prostate cancer: a report from the ASCENT Investigators. J.Clin.Oncol. 25 (6):669-674.

Berlin NI (2000) Treatment of the myeloproliferative disorders with 32P. Eur.J.Haematol. 65 (1):1-7.

Berthold DR et al (2008) Docetaxel plus prednisone or mitoxantrone plus prednisone for advanced prostate cancer: updated survival in the TAX 327 study. J.Clin.Oncol. 26 (2):242-245.

Berthold DR, Sternberg CN, and Tannock IF (2005) Management of advanced prostate cancer after first-line chemotherapy. J.Clin.Oncol. 23 (32):8247-8252.

Blake GM et al (1986) Sr-89 therapy: strontium kinetics in disseminated carcinoma of the prostate. Eur.J.Nucl.Med. 12 (9):447-454.

Boyle BJ et al (2001) Insulin-like growth factor binding protein-3 mediates 1 alpha,25dihydroxyvitamin $\mathrm{d}(3)$ growth inhibition in the LNCaP prostate cancer cell line through p21/WAF1. J.Urol. 165 (4):1319-1324.

Briggs C et al (2004) Assessment of an immature platelet fraction (IPF) in peripheral thrombocytopenia. Br.J.Haematol. 126 (1):93-99.

Bubley GJ et al (1999) Eligibility and response guidelines for phase II clinical trials in androgen-independent prostate cancer: recommendations from the ProstateSpecific Antigen Working Group. J.Clin.Oncol. 17 (11):3461-3467.

Buchan RC and Brindle JM (1970) Radioiodine therapy to out-patients - the contamination hazard. Br.J.Radiol. 43 (511):479-482.

Carducci MA et al (2003) Effect of endothelin-A receptor blockade with atrasentan on tumor progression in men with hormone-refractory prostate cancer: a randomized, phase II, placebo-controlled trial. J.Clin.Oncol. 21 (4):679-689.

Chapman CR and Gavrin J (1999) Suffering: the contributions of persistent pain. Lancet 353 (9171):2233-2237.

Cheung A and Driedger AA (1980) Evaluation of radioactive phosphorus in the palliation of metastatic bone lesions from carcinoma of the breast and prostate. Radiology 134 (1):209-212.

Coebergh, J. W. W., van de Poll-Franse, L. V., and Alers, J. C. Kanker in Nederland, Trends, prognoses en implicaties voor zorgvraag. Amsterdam. KWF , 65-73. 2004. 
Coleman RE et al (2005) Predictive value of bone resorption and formation markers in cancer patients with bone metastases receiving the bisphosphonate zoledronic acid. J.Clin.Oncol. 23 (22):4925-4935.

Cook RJ et al (2006) Markers of bone metabolism and survival in men with hormonerefractory metastatic prostate cancer. Clin.Cancer Res. 12 (11 Pt 1):3361-3367.

Dahut WL et al (2004) Randomized phase II trial of docetaxel plus thalidomide in androgenindependent prostate cancer. J.Clin.Oncol. 22 (13):2532-2539.

de Klerk JM et al (1994) Evaluation of thrombocytopenia in patients treated with rhenium186-HEDP: guidelines for individual dosage recommendations. J.Nucl.Med. 35 (9):1423-1428.

de Klerk JM et al (1992) Pharmacokinetics of rhenium-186 after administration of rhenium186-HEDP to patients with bone metastases. J.Nucl.Med. 33 (5):646-651.

DePuy V et al (2007) Effects of skeletal morbidities on longitudinal patient-reported outcomes and survival in patients with metastatic prostate cancer. Support.Care Cancer 15 (7):869-876.

Dijkman GA and Debruyne FM (1996) Epidemiology of prostate cancer. Eur.Urol. 30 (3):281295.

Etches RC (1999) Patient-controlled analgesia. Surg.Clin.North Am. 79 (2):297-312.

Fayers, P. M., Aaronson, N. K., Bjordal, K., Groenvold, M., Curran, D., and Bottomley, A. The EORTC QLQ-C30 Scoring Manual. 3rd ed. Brussels: European Organization for Research and Treatment of Cancer . 2001. Brussels. Ref Type: Generic

Figg WD et al (1996) Lack of correlation between prostate-specific antigen and the presence of measurable soft tissue metastases in hormone-refractory prostate cancer. Cancer Invest 14 (6):513-517.

Finlay IG, Mason MD, and Shelley M (2005) Radioisotopes for the palliation of metastatic bone cancer: a systematic review. Lancet Oncol. 6 (6):392-400.

Han SH et al (2002) The PLACORHEN study: a double-blind, placebo-controlled, randomized radionuclide study with (186)Re-etidronate in hormone-resistant prostate cancer patients with painful bone metastases. Placebo Controlled Rhenium Study. J.Nucl.Med. 43 (9):1150-1156.

Hartsell WF et al (2005) Randomized trial of short- versus long-course radiotherapy for palliation of painful bone metastases. J.Natl.Cancer Inst. 97 (11):798-804.

James ND et al (2008) Safety and Efficacy of the Specific Endothelin-A Receptor Antagonist ZD4054 in Patients with Hormone-Resistant Prostate Cancer and Bone Metastases Who Were Pain Free or Mildly Symptomatic: A Double-Blind, Placebo-Controlled, Randomised, Phase 2 Trial. Eur.Urol.

John SS et al (2008) Newer imaging modalities to assist with target localization in the radiation treatment of prostate cancer and possible lymph node metastases. Int.J.Radiat.Oncol.Biol.Phys. 71 (1 Suppl):S43-S47.

Jung K et al (2004) Comparison of 10 serum bone turnover markers in prostate carcinoma patients with bone metastatic spread: diagnostic and prognostic implications. Int.J.Cancer 111 (5):783-791.

Kaasa $S$ et al (2006) Prospective randomised multicenter trial on single fraction radiotherapy $(8 \mathrm{~Gy} \times 1)$ versus multiple fractions $(3 \mathrm{~Gy} \times 10)$ in the treatment of painful bone metastases. Radiother.Oncol. 79 (3):278-284. 
Kelly WK and Scher HI (1993) Prostate specific antigen decline after antiandrogen withdrawal: the flutamide withdrawal syndrome. J.Urol. 149 (3):607-609.

Klotz L (2008) Maximal androgen blockade for advanced prostate cancer. Best.Pract.Res.Clin.Endocrinol.Metab 22 (2):331-340.

Ko YJ et al (2001) A multi-institutional phase ii study of SU101, a platelet-derived growth factor receptor inhibitor, for patients with hormone-refractory prostate cancer. Clin.Cancer Res. 7 (4):800-805.

Krishnamurthy GT and Krishnamurthy S (2000) Radionuclides for metastatic bone pain palliation: a need for rational re-evaluation in the new millennium. J.Nucl.Med. 41 (4):688-691.

Lam MG et al (2009a) (188)Re-HEDP combined with capecitabine in hormone-refractory prostate cancer patients with bone metastases: a phase I safety and toxicity study. Eur.J.Nucl.Med.Mol.Imaging 36 (9):1425-1433.

Lam MG et al (2007) Combined use of zoledronic acid and 153Sm-EDTMP in hormonerefractory prostate cancer patients with bone metastases. Eur.J.Nucl.Med.Mol.Imaging.

Lam MG et al (2009b) Radiation safety considerations for the bone seeking radiopharmaceuticals. $89 \mathrm{SrCl} 2,186 \mathrm{Re}-\mathrm{HEDP}$ and 153Sm-EDTMP. Nuklearmedizin 48 (1):37-43.

Langley RE et al (2008) Early hormonal data from a multicentre phase II trial using transdermal oestrogen patches as first-line hormonal therapy in patients with locally advanced or metastatic prostate cancer. BJU.Int. 102 (4):442-445.

Lein $M$ et al (2007) Serial markers of bone turnover in men with metastatic prostate cancer treated with zoledronic Acid for detection of bone metastases progression. Eur.Urol. 52 (5):1381-1387.

Lewiecki EM (2006) RANK ligand inhibition with denosumab for the management of osteoporosis. Expert.Opin.Biol.Ther. 6 (10):1041-1050.

Lewington VJ et al (1991) A prospective, randomised double-blind crossover study to examine the efficacy of strontium-89 in pain palliation in patients with advanced prostate cancer metastatic to bone. Eur.J.Cancer 27 (8):954-958.

Lipton A et al (2008) Normalization of bone markers is associated with improved survival in patients with bone metastases from solid tumors and elevated bone resorption receiving zoledronic acid. Cancer 113 (1):193-201.

Maxon HR, III et al (1991) Rhenium-186(Sn)HEDP for treatment of painful osseous metastases: results of a double-blind crossover comparison with placebo. J.Nucl.Med. 32 (10):1877-1881.

McQuay, H. J., Collins, S., Carroll, D., and Moore, R. A. Radiotherapy for the palliation of painful bone metastases. The Cochrane Database of Systemic Reviews Issue 4, 1-22. 2008.

Mithal NP, Needham PR, and Hoskin PJ (1994) Retreatment with radiotherapy for painful bone metastases. Int.J.Radiat.Oncol.Biol.Phys. 29 (5):1011-1014.

Nam S et al (2005) Action of the Src family kinase inhibitor, dasatinib (BMS-354825), on human prostate cancer cells. Cancer Res. 65 (20):9185-9189.

Nilsson S et al (2005) Palliation of bone pain in prostate cancer using chemotherapy and strontium-89. A randomized phase II study. J.Pain Symptom.Manage. 29 (4):352-357. 
Oosterhof GO et al (2003) Strontium(89) chloride versus palliative local field radiotherapy in patients with hormonal escaped prostate cancer: a phase III study of the European Organisation for Research and Treatment of Cancer, Genitourinary Group. Eur.Urol. 44 (5):519-526.

Palmedo H et al (2003) Repeated bone-targeted therapy for hormone-refractory prostate carcinoma: tandomized phase II trial with the new, high-energy radiopharmaceutical rhenium-188 hydroxyethylidenediphosphonate. J.Clin.Oncol. 21 (15):2869-2875.

Piffanelli A et al (2001) Radionuclide therapy for painful bone metastases. An Italian multicentre observational study. Writing Committee of an Ad Hoc Study Group. Q.J.Nucl.Med. 45 (1):100-107.

Porter AT et al (1993) Results of a randomized phase-III trial to evaluate the efficacy of strontium-89 adjuvant to local field external beam irradiation in the management of endocrine resistant metastatic prostate cancer. Int.J.Radiat.Oncol.Biol.Phys. 25 (5):805-813.

Price DT et al (2002) Comparison of [18 F]fluorocholine and [18 F]fluorodeoxyglucose for positron emission tomography of androgen dependent and androgen independent prostate cancer. J.Urol. 168 (1):273-280.

Quilty PM et al (1994) A comparison of the palliative effects of strontium-89 and external beam radiotherapy in metastatic prostate cancer. Radiother.Oncol. 31 (1):33-40.

Quirijnen JM et al (1996) Efficacy of rhenium-186-etidronate in prostate cancer patients with metastatic bone pain. J.Nucl.Med. 37 (9):1511-1515.

Rago R (1998) Management of Hormone-Sensitive and Hormone-Refractory Metastatic Prostate Cancer. Cancer Control 5 (6):513-521.

Ricci S et al (2007) Clinical benefit of bone-targeted radiometabolic therapy with 153SmEDTMP combined with chemotherapy in patients with metastatic hormonerefractory prostate cancer. Eur.J.Nucl.Med.Mol.Imaging 34 (7):1023-1030.

Roos DE et al (2005) Randomized trial of 8 Gy in 1 versus 20 Gy in 5 fractions of radiotherapy for neuropathic pain due to bone metastases (Trans-Tasman Radiation Oncology Group, TROG 96.05). Radiother.Oncol. 75 (1):54-63.

Roque i Figuls, M., Martinez-ZApata, M. J., Alonso-Coello, P., Catala, E., Garcia, J. L., and Ferrandiz, M. Radioisotopes for metastatic bone pain. The Cochrane Database of Systemic Reviews Issue 4, 1-36. 2008.

Saad F et al (2002) A randomized, placebo-controlled trial of zoledronic acid in patients with hormone-refractory metastatic prostate carcinoma. J.Natl.Cancer Inst. 94 (19):14581468 .

Saarto $\mathrm{T}$ et al (2002) Palliative radiotherapy in the treatment of skeletal metastases. Eur.J.Pain 6 (5):323-330.

Sabbatini P et al (1999) Prognostic significance of extent of disease in bone in patients with androgen-independent prostate cancer. J.Clin.Oncol. 17 (3):948-957.

Sartor O et al (2007) Safety and efficacy of repeat administration of samarium Sm-153 lexidronam to patients with metastatic bone pain. Cancer 109 (3):637-643.

Sartor O et al (2004) Samarium-153-Lexidronam complex for treatment of painful bone metastases in hormone-refractory prostate cancer. Urology 63 (5):940-945. 
Scher HI et al (2008) Design and end points of clinical trials for patients with progressive prostate cancer and castrate levels of testosterone: recommendations of the Prostate Cancer Clinical Trials Working Group. J.Clin.Oncol. 26 (7):1148-1159.

Sciuto R et al (2001) Metastatic bone pain palliation with 89-Sr and 186-Re-HEDP in breast cancer patients. Breast Cancer Res.Treat. 66 (2):101-109.

Sciuto R et al (2002) Effects of low-dose cisplatin on 89Sr therapy for painful bone metastases from prostate cancer: a randomized clinical trial. J.Nucl.Med. 43 (1):79-86.

Sciuto R et al (1996) Radiosensitization with low-dose carboplatin enhances pain palliation in radioisotope therapy with strontium-89. Nucl.Med.Commun. 17 (9):799-804.

Serafini AN et al (1998) Palliation of pain associated with metastatic bone cancer using samarium-153 lexidronam: a double-blind placebo-controlled clinical trial. J.Clin.Oncol. 16 (4):1574-1581.

Smaletz O et al (2002) Nomogram for overall survival of patients with progressive metastatic prostate cancer after castration. J.Clin.Oncol. 20 (19):3972-3982.

Small EJ et al (2000) Prospective trial of the herbal supplement PC-SPES in patients with progressive prostate cancer. J.Clin.Oncol. 18 (21):3595-3603.

Small EJ et al (2004) Antiandrogen withdrawal alone or in combination with ketoconazole in androgen-independent prostate cancer patients: a phase III trial (CALGB 9583). J.Clin.Oncol. 22 (6):1025-1033.

Small EJ and Srinivas S (1995) The antiandrogen withdrawal syndrome. Experience in a large cohort of unselected patients with advanced prostate cancer. Cancer 76 (8):1428-1434.

Smeland S et al (2003) Role of strontium-89 as adjuvant to palliative external beam radiotherapy is questionable: results of a double-blind randomized study. Int.J.Radiat.Oncol.Biol.Phys. 56 (5):1397-1404.

Smith MR et al (2007) Predictors of skeletal complications in men with hormone-refractory metastatic prostate cancer. Urology 70 (2):315-319.

Sridhara R et al (1995) Evaluation of prostate-specific antigen as a surrogate marker for response of hormone-refractory prostate cancer to suramin therapy. J.Clin.Oncol. 13 (12):2944-2953.

Tannock I et al (1989) Treatment of metastatic prostatic cancer with low-dose prednisone: evaluation of pain and quality of life as pragmatic indices of response. J.Clin.Oncol. 7 (5):590-597.

Tannock IF et al (2004) Docetaxel plus prednisone or mitoxantrone plus prednisone for advanced prostate cancer. N.Engl.J.Med. 351 (15):1502-1512.

$\mathrm{Tu}, \mathrm{S}$. and Lin, S. Current trials using bone-targeting agents in prostate cancer. Cancer J. 14, 35-39. 2008.

Tu SM et al (2001) Bone-targeted therapy for advanced androgen-independent carcinoma of the prostate: a randomised phase II trial. Lancet 357 (9253):336-341.

Van der Linden YM et al (2004) Single fraction radiotherapy is efficacious: a further analysis of the Dutch Bone Metastasis Study controlling for the influence of retreatment. Int.J.Radiat.Oncol.Biol.Phys. 59 (2):528-537.

VIKC. Richtlijn 'diagnostiek en behandeling van pijn bij patiënten met kanker' Utrecht. 2008.

Visser, O. and van Noord, K. J. Feiten en fabels over kanker in Nederland. Utrecht. VIKC , 89. 2005.

VRO92. Min. VROM, Publicatiereeks Stralenbescherming nr. 1992/55, mei 1992. 
VROM. Recommendations: working with therapeutical doses of radionuclides, The Hague. 2005.

Wang C et al (2002) Reticulated platelets predict platelet count recovery following chemotherapy. Transfusion 42 (3):368-374. 


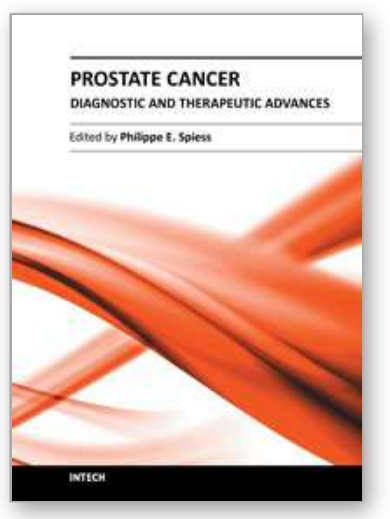

\author{
Prostate Cancer - Diagnostic and Therapeutic Advances \\ Edited by Dr. Philippe E. Spiess
}

ISBN 978-953-307-319-4

Hard cover, 378 pages

Publisher InTech

Published online 25, November, 2011

Published in print edition November, 2011

In this book entitled "Prostate Cancer - Diagnostic and Therapeutic Advances", we highlight many of the significant advances made in our treatment armamentarium of prostate cancer. The book is subdivided into four sections termed: 1) novel diagnostic approaches, 2) surgical treatments options, 3) radiation therapy and its potential sequelae, and 4) medical management and its treatment complications. After reading the present book, readers will be very familiar with the major clinical advances made in our multifaceted treatment approach to prostate cancer over the past decade. This book is a tribute to our pioneering urologists and allied healthcare professionals who have continually pushed forward our traditional therapeutic envelope.

\title{
How to reference
}

In order to correctly reference this scholarly work, feel free to copy and paste the following:

Marnix G.E.H. Lam and Bernard A. Zonnenberg (2011). Bone Seeking Radiopharmaceuticals for Metastatic Bone Pain, Prostate Cancer - Diagnostic and Therapeutic Advances, Dr. Philippe E. Spiess (Ed.), ISBN: 978953-307-319-4, InTech, Available from: http://www.intechopen.com/books/prostate-cancer-diagnostic-andtherapeutic-advances/bone-seeking-radiopharmaceuticals-for-metastatic-bone-pain

\section{INTECH}

open science | open minds

\section{InTech Europe}

University Campus STeP Ri Slavka Krautzeka 83/A 51000 Rijeka, Croatia Phone: +385 (51) 770447

Fax: +385 (51) 686166 www.intechopen.com

\section{InTech China}

Unit 405, Office Block, Hotel Equatorial Shanghai No.65, Yan An Road (West), Shanghai, 200040, China 中国上海市延安西路65号上海国际贵都大饭店办公楼 405 单元 Phone: $+86-21-62489820$

Fax: $+86-21-62489821$ 
(C) 2011 The Author(s). Licensee IntechOpen. This is an open access article distributed under the terms of the Creative Commons Attribution 3.0 License, which permits unrestricted use, distribution, and reproduction in any medium, provided the original work is properly cited. 